\title{
CONCEPTO DE FILOSOFÍA DE LA EDUCACIÓN
}

\author{
Josep M. ${ }^{a}$ QUINTANA CABANAS
}

La práctica de la Pedagogía es tan antigua como la cultura literaria; sin embargo, el estudio de la Pedagogía como ciencia, es decir, en cuanto disciplina científica, es relativamente reciente, pudiendo señalarse su inicio en 1806, por obra de Herbart. Otro tanto podríamos decir de la Filosofía de la educación: como serie de reflexiones filosóficas en torno al hecho educativo, ha tenido lugar desde que se ha escrito Pedagogía, pues tanto filósofos como pedagogos han dicho algo al respecto; pero como ciencia autónoma dentro de las ramas del saber, tanto pedagógico como filosófico, y, más concretamente, como disciplina académica en los $c u$ rricula de estudios, su existencia ya no es tan clara. Tal vez podría decirse que la Filosofía de la educación, en este último sentido, está aún por hacer. Es cierto que existen interesantes aportaciones: numerosos autores, en efecto, nos han ofrecido obras que se nos presentan bajo el título de Filosofía de la educación; pero en general resultan obras parciales, tanto por los temas tratados como, a veces, por su enfoque, y queda pendiente una obra enciclopédica y sistemática que ofrezca un edificio completo, fundamentado y armónico de todo lo que cabe considerar cuando hablamos de la Filosofía de la educación.

Esto ha sucedido, sin duda, por falta de especialistas dedicados a este campo, ya que han de ser personas dotadas de una formación muy particular. El mero pedagogo no sirve para ello, pues le faltan los conocimien. tos filosóficos requeridos. La Filosofía de la educación es Filosofía y, como tal, es quehacer privativo del filósofo. Pero ocurre que es un campo muy concreto de la Filosofía, que precisa de especiales conocimientos pedagógicos y, que por no haberlos recibido, los filósofos normalmente no se dedican a este campo, que les resulta extraño. En resumen, la Filosofía de la educación como disciplina científica ha sido tierra de nadie, $y$ ha quedado por configurar.

Pero el actual desarrollo de los curricula universitarios exige que esta laguna sea colmada. En muchas universidades se explica y estudia Filosofía de la educación, y debe hacerse de un modo cabal; y en los lugares donde esta asignatura no consta en los planes de estudio, habría de introducirse sin demora. La Filosofía de la educación como tema intelectual ha tenido siempre algunos grandes cultivadores; pero ha llegado el mo. mento de que encuentre, además, su identidad científica y académica. Las consideraciones que siguen quisieran ayudar a esa labor.

\section{LA FILOSOFÍA DE LA EDUCACIÓN DENTRO DEL CUADRO DE LA FILOSOFÍA EN GENERAL}

Para J. Piaget «la filosofía consiste en una búsqueda de lo absoluto o en un análisis de la totalidad de la experiencia humana ${ }^{1}$. El filósofo la hace de una manera sistemática y profunda, pero ningún hombre deja de hacerla, aunque sea a un nivel superficial y poco consciente; al ser el hombre racional, siempre elabora una razón de ser de las cosas y una justificación de sus actos, con lo cual no puede evitar el filosofar. Esto ocurre particularmente en actividades humanas por antonomasia, como es la de la educación: en ella se trata de la formación de un tipo de persona y de una configuración de valores; educar es poner en juego una determinada filosofía. Debajo de toda teoría de la educación subyace una filosofía de la educación.

Mejor será, por consiguiente, que -contra la costumbre habitual- esa filosofía de la educación se haga explícita y crítica. Esto equivale a sentar la necesidad de una Filosofía de la educación, no sólo desde el requerimiento de su inevitabilidad, sino también desde el de la trascendencia que tiene en la orientación direccional que tomará la edu* cación. Creemos que no es preciso insistir en esa tesis evidente, pero -como contrapartida a lo que suele suceder en nuestros ambientes pedagógicos bueno será recordar que los autores han visto así esta cuestión; desde $O$. Reboul, para quien "un maestro que no se haga preguntas sobre lo que ense. ña desprecia sus asignaturas, la cultura, a sus

1 Piaget, J., Epistemología de las ciencias humanas, Buenos Aires, 1972, Proteo, p. 172. 
alumnos y a sí mismo" ${ }^{2}$, hasta $\mathrm{Fr}$. De Honvre y R. Hubert, el cual escribe: "Una Pedagogía, es especialmente en su parte general, que traza las líneas directivas en las que debe encarrilarse la acción educativa, es ante todo una filosofía de la educación [...]. Desde que llegamos a la conciencia y a su acción, estamos en los dominios de la Filosofía. La doctrina de la educación no sólo la presupone, sino que la completa y es, como la moral, su puesta en acción, su necesario acabamierito" ${ }^{4}$. Podríamos seguir mencionando textos de J. Mantovanis o de G. Gusdorfo, pero preferimos éste de E. Spranger: "Quien construye una Pedagogía que excluye la me tafísica de raíz, no llega a ver ni por asomo el fenómeno pedagógico en su origen y esenciar?.

Eso demuestra que la Pedagogía es necesariamente filosófica. Pero hay más: la Filo. sofía es necesariamente pedagógica. La $\mathrm{Fi}$ losofía, en efecto, está para orientar al hom. bre; no quiere quedarse en meras especula* ciones, sino convertirse en acción. Mas en este último paso, la Filosofía deja de ser tal para convertirse en Pedagogía. La Pedagogía no es otra cosa que Filosofía puesta en práctica; por eso, según Dilthey, «la última palabra del filósofo es la Pedagogía, pues todo especular se realiza por el obrar»; "des" de un punto de vista general, la floración y fin de toda verdadera Filosofía es la Pedagogía en su más amplio sentido: teoría de la formación del hombre" ${ }^{8}$. La Pedagogía es el coronamiento y consumación de la Filosofía.

La ręalidad y necesidad de una Filosofía de la educación queda, pues, fuera de duda.

2 REBOUL. O., ¿Transformar la suciedad? ¿Transformar la educación?, Madrid, 1972, Narcea, p. 70.

3 DE Houvre, $F$., aEs un hecho muy de notat que las grandes batallas pedagógicas se libran siempre fuera del campo de la psicología pedagógica, y aun fuera del campo de la Pedagogía propiamence dichas. Ensayo de Filosofia Pedagógica, Madrid, 1932, Razón y fe, p. 13.

4 Huberi, R., Tratado de Pedagogia General, Buenos Aires, 1963, El Ateneo, p. 24.

5 Cf. Mantovani, J., Educación y plenitud bumana, Buenos Aires, 1957, El Ateneo, p. 16.

6 GusDORF. G, ¿ ¿Para qué los profesores?, Madtid, 1969. Edicusa, pp. 84, 291, 292, exc.

7 SPRANGER, E., «Vom Wissenschaftcharakter der Päda. gogik», en HARTMANN, G., Beiträge zur Einheit von Bildung und Sprache in geistigen Sein, Berlin, 1957, p. 10.

8 DitTHEY, W., Historia de la Pedagogia, Buenos Aites, 1968, Losada, p. 11.
Lo que no resulta tan claro es la naturaleza de este tipo de saber. Ligado como está a la Filosofía -es decir, siendo Filosofía- le ocurre lo que a ésta: es un saber problemático. La Filosofía, en efecto, no tiene una definición clara de su concepto, ni de la determinación de su método, ni del establecimiento de sus partes constitutivas, ni del alcance que consiguen sus logros; y esto mismo es lo que ocurre en Filosofía de la educación. De hecho, no hay una sola Filosofía de la Educación sino muchas, cada una de ellas con la pretensión de ser verdadera y absoluta: mientras el racionalismo le da una base metafísica, el neopositivismo quiere reducirla a cuestiones de lenguaje'; y si el idealismo parte de una concepción del hombre como espíritu libre y creativo, el empirismo lo ve como un ser abocado a superar unas determinadas condiciones de vida. Esto no debe desalentarnos, sino invitarnos a profundizar más en la Filosofía de la educación, dentro de nuestro interés por la Filosofía en general.

Presentándose la primera dentro del cua" dro de esta última, hemos de establecer dicho cuadro para localizar la Filosofía de la educación en el puesto que en el mismo le corresponde, y ver las relaciones que guarda con la Filosofía en general y con las diversas ramas de la misma en particular. Son varias las clasificaciones que se han elaborado en cuestión de materias filosóficas; nosotros proponemos una más, con la esperanza de que sirva para aclarar precisamente el puesto y la función que corresponden a la Filoso. fía de la educación dentro de todo el sistema de especialidades filosóficas. Para simplificar, reducimos nuestra taxonomía a las materias clásicas o fundamentales, y lo nuevo es sólo alguno de los criterios en que se funda, como comentaremos en seguida. Dicha clasificación es la siguiente.

\section{DIVISIÓN DE LA FILOSOFIA}

\section{Filosoffa propedéutica (o preparatoria) \\ - Lógica clásica y matemática. \\ - Metodología. \\ - Filosofía del lenguaje.}

9 Véaste por ejemplo, entre otras obras, SMITH, B. O 
2. Filosofía general (cuestiones básicas de Filosofía)

2.1. Filosofía teorética (comprensión de la realidad)

2.1.1. Metafísica (estudio del ser)

-Ontología (estudio del ser en general).

- Teodicea (esrudio del ser absoluto).

- Epistemología (estudio del co. nocimiento).

2.1.2. Filosofía natural (estudio de los entes existentes)

- Cosmología (estudio de los constitutivos cósmicos).

- Antropologia (estudio del hombre).

2.2. Filosofía práctica (estudio de las normas de comportamiento): Ética

3. Filosofta especial (ámbitos concretos de Filosofáa: consideración filosófica de ámbitos concretos de la realidad)

- Filosofía de la Historia.

- Estética.

- Filosofía social.

- Axiología.

- Historia de la Filosofía.

4. Filosofía aplicada (aplicación de las teorías filosóficas a los problemas prácticos de la vida)

$\rightarrow$ Filosofía de la ciencia (Epistemología)

- Filosofía del derecho.

- Filosofía de la religión.

- Filosofía de la educación.

\section{LA FILOSOFÍA DE LA EDUCACIÓN COMO UNA FILOSOFÍA APLICADA}

La originalidad de esta clasificación estriba en incorporar a la división de la Filosofía las categorías epistemológicas de ciencia "general» ciencia "especial» y ciencia "aplicada". Lo hacemos sin ningún reparo, amparados en la idea de que la ciencia no es un concepto unívoco sino analógico, de tal manera que, no habiendo un solo modo de concebir la ciencia, esta denominación conviene también, en cierto modo, a la Filosofía, que tiene con las ciencias algún rasgo común, sobre todo el de la racionalidad en el conocimiento.

y ENNIS, R. H., Lenguaje y conceptós en la educación, Bue. nos Aires, 1970, El Ateneo
Ahora bien, es frecuente y provechoso el hecho de dividir una ciencia en teorética y aplicada, y la primera, en general y especial. La ciencia "teorética" (o "pura») es la que estudia desinteresadamente su objeto, por afán de mero conocimiento intelectual de la realidad: es "general» si estudia los aspectos fundamentales y genéricos de ese objeto, y es "especial» cuando se ocupa de las áreas regionales del mismo. La ciencia "aplicada", en cambio, es interesada, y en ella el conocimiento no es un fin en sí, sino sólo un medio para resolver dificultades prácticas de la vida; no amplía el campo de conocimientos (que sólo queda ampliado por la ciencia teoréti$\mathrm{ca}$, tanto general como especial), sino que se limita a utilizar los conocimientos adquiri* dos por esa última y a aplicarlos a los proble. mas de la existencia humana.

Este modo de clasificar las ramas de una ciencia resulta cómodo y adecuado en numerosos tipos de ciencias, como la Química, la Medicina o la Sociología; ofrecemos el ejemplo de la Psicología a modo de ilustración:

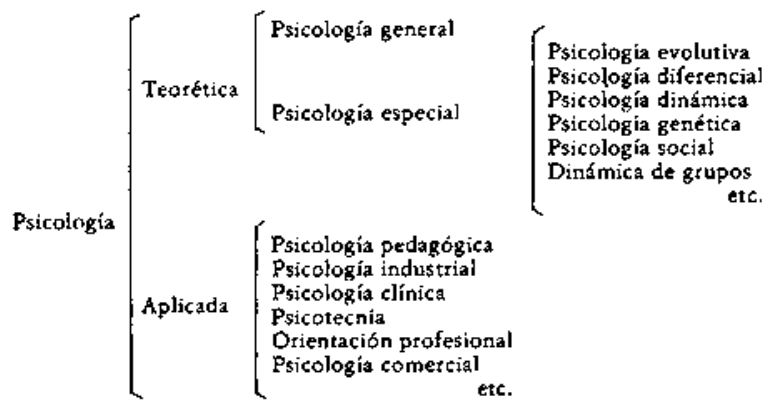

También la Filosofía se puede encuadrar en ese esquema epistemológico, aun cuando nunca se había pensado en utilizarlo. Sucede, en efecto, que aparte de unas ramas propedéuticas - que no serían Filosofía strictu sensu, aun cuando supongan también cuestiones filosóficas (en realidad todo tipo de saberes implican temas filosóficos)- el cuerpo principal de ramas filosóficas es susceptible de dividirse en ramas generales, especia. les y aplicadas. El criterio para considerarlas en uno u otro grupo es el que acabamos de exponer, como en cualquier ciencia. 
El término "Filosofía general» no es nuevo en Filosofía. En Alemania, por ejemplo, el currículum universitario de Filosofía comprende la "Allgemeine Philosophie», que es exactamente eso. En nuestro país, en cambio, no se conoce esta expresión ni esta asignatura que, en caso de existir, se discutiría si debe entenderse como "Filosofía en general" (correspondiendo a los clásicos manuales de Filosofía que constituyen un compendio de todo el saber filosófico) o como "Fin losofía general", es decir, fundamental, que se ocupa sólo de las cuestiones troncales, postura esta última que nos parece más aceptable ${ }^{10}$. Ha habido alguna propuesta más o menos vaga en este sentido, como la de Ardigò ${ }^{11}$. Nosotros llamamos Filosofía general a todo el corpus básico de Filosofía.

La Filosofía "especial» - de acuerdo con los criterios expuestos- apenas difiere cualitativamente de la Filosofía "general», siendo su diferencia más bien de tipo cuantitativo, al especificarse por un mero criterio de regionalidad en la circunscripción de su objeto de estudio, que es análogo al de la Filosofía general, con la sola distinción de refe. rirse a aspectos más concretos, algo margi* nales (cuestión ésta muy relativa, determinada a menudo por factores puramente históricos y accidentales; teóricamente, en efecto, sería muy difícil querer justificar, por ejemplo, que la Cosmología pertenece a la Filosofía general, mientras que la Estética pertenece a la especial, pues tanto el cos. mos, como la belleza o la historia han de ser

10 Una polémica análoga la conocemos, por ejemplo, en Pedagogia, donde hoy día se discute la naturaleza y existencia de una Pedagogia general, que muchos creen disuelta en las llamadas «Ciencias de la Educación». Frence a esta opinión, nosotros hemos reivindicado el nombre y el contenido de una Pedagogía general.

11 En 1884 Ardigò acuñó el técmino Peracología (del griego pêras, pératos, punto esencial) para indicar la parte general de la Filosofia, es decir, la parte que estudia las cuestiones fundamentales al margen de los campos patticulares de las disciplinas filosóficas aespeciales», o sea, la Psicología y la Sociología. El reparo que tenemos que hacer a este autor es que estas dos últimas materias no son filosóficas ni constituyen, por consiguiente, tamas de la Filosofía especial», con lo cual su Peratología o Filosofia egeneral» se confunde con la Filosofía simpliciter. considerados, por su mismo título, como aspectos de la realidad).

La Filosofía "aplicada", en cambio, ya es otro asunto, pues, según se dijo, sus ramas no constituyen ámbitos de estudio, sino que se limitan a aplicar, a problemas surgidos, los conocimientos elaborados por las ramas teóricas, generales o especiales. Así, por ejemplo, la Filosofía del Derecho utilizará principios de la Ética, de la Antropología y de la Filosofía social, y con esto y otros conocimientos (sacados de la Psicología, la Sociología, la Etología, etc.) tratará de responder a sus interrogantes; queremos decir que tales interrogantes no requieren un tipo de reflexión distinta a la efectuada en todas esas disciplinas, sino sólo un aprovechamiento de la misma.

Eso es precisamente lo que sucede en la Filosofía de la Educación, y por eso la con. sideramos como aplicada. A semejanza de lo que ocurre en Derecho, también en educación nos hallamos con una serie de pregun* tas ante las cuales necesitamos una respuesta: ¿Qué confianza podemos tenex en el desarrollo natural del educando? ¿En qué consiste ser libre? ¿Hay que educar para una trascendencia? ¿Son absolutos o relativos los valores? ¿Cuál es el modelo de personalidad perfecta?, y tantos otros interrogantes, nu" merosos y acuciantes. La Filosofía de la Edu. cación es precisamente la encargada de formular tales preguntas y organizar la reflexión encaminada a encontrar una respuesta; y para ello sólo tiene que aprovechar la refle. xión establecida por la Antropología, la Ética, la Axiología, la Metafísica, la Teodicea, la Estética y otros saberes que puedan contribuir a esclarecer los fines de la educación.

De este modo, la Filosofía de la Educación puede ser claramente clasificada como una rama de Filosofía "aplicada"; así la entienden algunos autores, aunque no le hayan atribuido esta categoría epistemológica ${ }^{12}$.

12 Veamos un par de textos que corresponden a semejante concepción. D. J. O'Connor: "Hasta tanto no se esclarezca nuestra mente con respecto a la naturaleza de la Filosofía, no será posible adoptar una posición clara con respecto a los planteos de Filosofía de la educación $[\ldots .$.$] . No existe una materia denominada Filo.$ 
Pero con decir esto no basta. Pues ocurre igualmente que, por otro lado, la Filosofía de la Educación (es decir, una parte de ella, de su contenido) debe ser considerada como Filosofía «especial». ¿Cómo es esto posible? Lo es desde el momento en que se propone estudiar filosóficamente el ser de la educa* ción, su naturaleza entitativa. La educación, en efecto, es un fenómeno real, una parte de la realidad (como lo son la historia, la belleza o la vida social), y puede ser considerada bajo el prisma filosófico. Ésta ha sido la pos" tura clásica entre los que han hecho Filosofía de la educación, y de ellos tenemos en España representantes bien conocidos: no sólo los que se han limitado a hacer una Ontología aristotélico-tomista de la educación $n^{13}$, sino también otros que la han elabo. rado según unos esquemas más amplios y actuales, como A. Sancristóbal ${ }^{14}$. También se sitúa en esta línea epistemológica $R$. Nassif cuando entiende la Filosofía de la educa ción (o «Filosofía pedagógica», según otra

sofía de la educación, así como no existe una materia denominada Filosofía de la ciencia [...]. Existen problemas de Filosofia de gran importancia que interesan a los estudiosos de la educación, así como problemas de Filosofia que interesan especialmente a los investigadores científicos», AI adentranos más profundamen. te en los problemas de la Filosofía de la educación, llegan éstos a convertirse en los problemas tradiciona. les de la Filosofias. (Introducción a la Filosofia de la educa. ción, Buenos Aires, Paidós, 1971, pp. 1995 y 201). M. J. ADLER: aLos problemas últimos de la educación, como los del derecho y la medicina, son prácticos. Son cuestiones sobre lo que deberá hacerse para educar a un hombre: sea uno mismo u otro ser. Esto no significa, naturalmente, que no puedan presentarse cuesziones puramente teóricas sabre la educación [...]. Los priacipios genetales en que se fundan los procedimientos educativos constituyen la Filosofía de la educación, como una serie de respuescas a las cuestiones práccicas más generales que pueden contestarse sobre lo que se debe hacer pedagógicamente». ( $\alpha$ En defensa de la Filosofia de la educación», en KILPATRICK. W. H. y otros Filosofia de la educación, Buenos Aires, 1946, Losada, P. 246).

13 GONZÄLEZ. ĀLVAREZ, A., Filotofía de la educaciön, Buenos Aires, 1967, Ttoquel; PACIOS, A., Ontología de lo educación, Madrid, 1954, C.S.I.C.; GARCíA Hoz, V., Cuestiones de Filosofia individual y social de la educación, Madrid, 1962, Rialp.

14 SAN CRISTÓbal Sebastian. A., Filosofía de la educación, Madrid, 1965, Rialp. expresión suya) como "Filosofía de la cultura»'s.

Por cierto que cabría discutir la conveniencia de usar la expresión "Filosofía de la educación" o bien "Filosofía pedagógica". Para muchos existe un caos en la actual ter. minología pedagógica, sobre todo desde que se ha puesto de moda la expresión «Ciencias de la Educación» arrinconando el viejo nombre de "Pedagogía". Sin embargo, nosotros tenernos eso bien claro, y en la propuesta que hicimos en el II Seminario de "Epistemología y Pedagogían (Salamanca, 1981), distinguimos entre "Ciencias de la Educación" y "Ciencias Pedagógicas" (las primeras son ciencias "especiales", que estudian el becbo "educacional"; y las segundas son ciencias "aplicadas", que aplican al acto "educativo" los conocimientos logrados por otras ciencias): la Filosofía de la educación debería ser llamada así por el hecho de que estudia el ser o naturaleza de la educación (actuando como una Filosofía "especial»), y habría que llamarla "Filosofía pedagógica» cuando actúa como una Filosofía "aplicada»; ciertamente, como Filosofía "aplicada» des. pliega una gran riqueza de temas tanto en número como en valiosas aportaciones para la actividad educativa, mientras que como Filosofía "especial" se reduce a unos pocos temas cuyo interés es sólo especulativo y abstracto.

Pese a esta distinción que creemos epistemológicamente válida y necesaria, pensamos que en terminología debemos apelar también a un criterio de comodidad y simplificación, y por eso nosotros usamos el nombre "Filosofía de la educación", cuyo uso se ha impuesto entre nosotros, para abarcar las dos categorías de temas a que hemos estado aludiendo.

1) Ricardo NASSIF: «La Filosofía de la educación puede definirse como el conjunto de reflexiones sobre el hecho y e] proceso educativos conectados con la totalidad de la cultura y de! hombre, al mismo tiempo que la conciencia de la unidad y la dirección del obrar educativo». «Este concepto general de Filosofía de la educación se apoya en el análisis de las máltiples definiciones e interpretaciones que de la mis. ma circulan en nuestros días, pero da preeminencia a la concepción de la Filosofía pedagógica como Filosofía de la cultura», Pedagogia general, Buenos Aires, 1958, Kapelusz., p. 74. 


\section{RELACIONES ENTRE «FILOSOFIA DE LA EDUCACIÓN» $Y$ "TEORĹA DE LA EDUCACIÓN"}

Un buen catalizadox de esa crisis de la epistemología pedagógica a la que varias veces hemos aludido, lo constituye la aparición de una nueva disciplina y un nuevo nombre curricular: la "Teoría de la Educación". ¿De qué tratará esa materia? Para algunos es patente: si ha desaparecido la clásica "Pedagogía general", la Teoría de la Educación viene a cubrir el hueco dejado por ella, y con aires de modernidad, pues supone una renovación temática y, sobre todo, presenta una imagen de progresismo, ya que lleva nombre de una "ciencia de la educación".

Cabría hacer largas consideraciones sobre esta nueva materia, que goza de simpatía universal; pero desde luego salta a la vista la notable relación que tiene con la Filosofía de la educación. Se habló ya en este sentido, desde otras coordenadas filosóficas -nque no podemos aceptar-, cuando J. Dewey, en su pragmatismo, concebía la Filosofía de la educación como una teoría general de la educación; y al igual que este autor, son bastantes hoy día los que, huyendo de toda especulación filosófica, rechazan una Filosofía de la educación y se contentan con poner, como sucedáneo suyo, una "Teoría de la Educación». Porque, quiérase o no, la Teoría de la Educación implica una problemática ideológica inseparable de la educación $\mathrm{y}$, por consiguiente, su parentesco con la Filosofía de la educación resulta insoslayable.

En cuanto a nosotros, por un lado admitimos tanto la Filosofía de la educación como la Pedagogía general y la Teoría de la Educación: lo único que debemos hacer es, en tal caso, señalar sus incumbencias y acotar los campos respectivos. Por otro lado, entendemos que la Teoría de la Educación puede y debe tener una doble dimensión: científica e ideológica; es decir, puede hacerse como ciencia positiva o dando cabida a reflexiones antropológicas y filosóficas. Creemos que, aun cuando se haga lo primero, también puede y debe hacerse lo segundo, con lo cual se entra ya en el campo propio de la Filosofía de la educación.

Bueno será que expongamos nuestro concepto de Teoría de la Educación. La definimos diciendo que "es la parte de la Pedagogía general que estudia lo esencial del acto educativo, a saber: su naturaleza y sus fines, los factores que lo constituyen y la parte que en el mismo han de tener el educando y el educadorm. A la Teoría de la Educación le compete estudiar qué es la educación fenomenológicamente, del mismo modo que la Filosofía de la educación ha de decirnos qué es esencialmente. Una y otra quieren explicar qué es la educación; pero mientras la Filosofía de la educación opera con presupuestos metafísicos (la comunicabilidad del saber, la perfectibilidad humana, las nociones de cualidad y hábito, etc.), la Teoría de la Educación abarca temas más inmediatos e intuitivos: el concepto de educación, el acto educativo, la relación educador-educando, los condicionamientos de la educación, sus límites, etc. También son propios de la Teoría de la Educación temas como los fines de la educación, las nociones de libertad-autoridad o los valores que podemos considerar como típicos de la Filosofía de la educación. En realidad, hay unos temas que aparecen como privativos de cada una de estas dos disciplinas y otros que, por el contrario, son comunes a ellas; entre estos últimos está, desde luego, el que estudia la naturaleza de la educación y el que atiende a sus bases antropológicas.

A modo de ilustración (y con pretensiones sólo improvisadas e indicativas, y de ninguna manera exhaustivas), ofrecemos el siguiente esquema, en el que constan los principales campos propios de la Teoría de la Educación y de la Filosofía de la educación, y que muestra tanto los ámbitos diferenciales como los comunes.

La Filosofía de la Educación se ocupa sólo de aspectos esenciales de la Educación, mientras que la Teoría de la Educación recoge aspectos más existenciales o circunstanciales. La segunda quiere "explicar" qué es la educación y el educar, mientras que la primera, además de esto (aspecto que tiene en común con la Teoría de la Educación, y 


\begin{tabular}{|l|l|}
\hline $\begin{array}{l}\text { Concepción del mundo y de la vida } \\
\text { Fines de la educación; la perfecti- } \\
\text { bilidad humana } \\
\text { Los valores y la educación } \\
\text { Etica y educación } \\
\text { El derecho y el deber de educar } \\
\text { La filosofía de los sistemas pedagógicos }\end{array}$ & $\begin{array}{l}\text { Filosofía de } \\
\text { la Educación }\end{array}$ \\
\cline { 2 - 3 } & $\begin{array}{l}\text { La Antropología en la educación } \\
\text { Aspecto filosófico del proceso } \\
\text { educativo }\end{array}$ \\
Teoría de & $\begin{array}{l}\text { Fenomenología del proceso educativo } \\
\text { Aspectos cibernéticos y sistémicos de la } \\
\text { educación } \\
\text { Teoría de la comunicación y educación } \\
\text { Aspectos psicológicos de la educación } \\
\text { Aspectos sociológicos de la educación } \\
\text { Aspectos históricos de la educación }\end{array}$ \\
\hline
\end{tabular}

que la constituye como una Filosofía "es" pecial»), pretende "fundamentar» filosóficamente la actividad educativa (hecho que la constituye como una Filosofía "aplicadas). En unos aspectos, la Filosofía de la educación forma parte plenamente de la Teoría de la Educación; pero en otros, tiene un campo relativamente suyo, pues se ocu. pa más o menos remotamente de elaborar unos fundamentos para la educación.

Sería interesante que pudiera hacerse una Teoría de la Educación científica; pero las bases científicas en que debería apoyarse son, hoy por hoy, tan exiguas que el pretender hacerla es cosa vana, y los intentos existen. tes no conducen a nada práctico. En general, se hace una Teoría de la Educación de tipo ideológico; pero entendemos que esto tiene lugar no como mero sucedáneo de una teoría científica, sino como exigencia de los problemas considerados: de tal modo que, aun cuando hubiera una amplia teoría científica de la educación, la teoría ideológica tendría también plena vigencia, justificación y oportunidad.

\section{CONCEPTO Y CONTENIDO DE LA FILOSOFÍA DE LA EDUCACIÓN}

Todo lo dicho anteriormente quedará más claro si tratamos de especificar mejor los contenidos de la Filosofía de la educación, es decir, su temática. Hace falta insistir en ello, pues la mayoría de tratados de Filosofia de la educación resultan incompletos, ya que se limitan a desarrollar uno o algunos temas, y dejan por considerar otros que también son importantes. A este hecho aludíamos al comienzo, cuando afirmábamos que la Filosofía de la educación, como disciplina cien. tífica o académica, se halla todavía en perío. do de gestación.

De acuerdo con todo lo que hemos dicho, creemos que un temario completo de Filosofía de la educación ha de abarcar diversas áreas. Como muestra de lo que esto podría ser, ofrecemos a continuación el temario confeccionado por nosotros y explicado en Ia Universidad de Barcelona, durante los cuxsos 1972-1978, y que puede servir de orientación: 


\section{Introducción}

1. La función de la Filosofía en la vida humana

2. La Filosofía de la educación

II. La concepción del mundo y de la vida

3. Principales cosmovisiones, como bases para determinar el ideal educativo

4. El naturalismo

III. La concepción del bombre

5. La naturaleza humana

6. Soluciones históricas al problema del hombre

7. El concepto de hombre en autores diversos

8. El destino del hombre

9. Sociedad e individuo

10. La familia y su papel educador

IV. Los valores y la educación

11. Los valores en la educación

12. La Ética en la educación

13. El deber en la conducta personal

14. Los principales modos de concebir la moral

15. Corrientes éticas contemporáneas

16. Religiosidad y educación

17. La educación sexual

V. La concepción de la educación

18. Naturaleza y posibilidad de la educación
19. La eđucación como juego de antinomias

20. Los fines de la educación

21. La libertad en el hombre; el autodesarrollo

22. La antinomia libertad-autoridad en educación

23. El derecho y la obligación de educar.

En el área $V$, que estudia el ser de la educación, los temas 18,19 y 22 son de Filosofía "especial» de la educación; todos los otros puntos del temario, en cambio, suponen una Filosofía de la educación como Filosofía "aplicada».

Creemos que la aportación más notable de este temario es la explicitación de unas áreas, ninguna de las cuales puede ser olvidada; la especificación de los temas incluidos es ya más aleatoria, pues podrían añadirse temas, como los referentes a la educación estética, política y económica.

Estimamos que en esta construcción temática es donde mejor puede quedar reflejado lo que hemos intentado explicar, a saber, un concepto comprensivo de lo que es la Filosofía de la Educación, que podríamos resumir en la siguiente definición: "Filosofía de la educación es la explicación filosófica de la educación, y la elaboración crítica de los principios ideológicos que presupone el acto educativo y que sirven para orientarlo" (la primera parte de la definición alude a una Filosofía de la educación "especial», y el resto a una Filosofía "aplicada»). 